\title{
Intervenir E Investigar En El Aula: Estrategia Didáctica Para Aprender Química
}

\author{
M.C. María Guadalupe Ruacho Soto \\ Dr. Rigoberto Marín Uribe \\ Dra. Isabel Guzmán Ibarra \\ Dra. Ma. Concepción Soto Valenzuela \\ Universidad Autónoma de Chihuahua, México
}

Doi:10.19044/esj.2021.v17n7p31

Submitted: 01 December 2020

Accepted: 13 February 2021

Published: 28 February 2021
Copyright 2021 Author(s)

Under Creative Commons BY-NC-ND 4.0 OPEN ACCESS

Cite As:

Guadalupe Ruacho Soto M., Marín Uribe R., Guzmán Ibarra I. \& Soto Valenzuela C. (2021). Intervenir e Investigar en el Aula: Estrategia Didáctica para Aprender Química. European Scientific Journal, ESJ, 17(7), 31. https://doi.org/10.19044/esj.2021.v17n7p31

\section{Resumen}

El propósito de este artículo es presentar resultados de la aplicación de una estrategia didáctica para aprender química en la educación superior sustentada en el Modelo para el Desarrollo y Evaluación de Competencias Académicas. El estudio se realizó con estudiantes de segundo semestre de la asignatura "Procesos químicos de la vida humana" de la Licenciatura en Nutrición. Se construyeron y aplicaron, en una intervención áulica, secuencias didácticas cuya estructura incluye dos dispositivos: uno de formación y otro de evaluación auténtica; el dispositivo de formación emplea situaciones problema reales de la profesión, cuya resolución comporta la movilización de saberes y el desarrollo de competencias. El proceso de intervención áulica incluyó momentos de práctica reflexiva y de acompañamiento docente. El enfoque de la investigación-formación-acción realizado incorpora el análisis cualitativo de datos recogidos mediante videograbación, cuaderno de notas y observación reflexiva de la intervención. Se concluye que la estrategia didáctica produce cambios graduales al transitarse de un estilo de docencia tradicionalista a una práctica educativa innovadora transformada y transformadora. 
Palabras-claves: Secuencias Didácticas, Situaciones Problema, Procesos Químicos, Didáctica De La Química

\title{
Intervene and Investigate in the Classroom: Didactic Strategy to Learn Chemistry
}

\author{
M.C. María Guadalupe Ruacho Soto \\ Dr. Rigoberto Marín Uribe \\ Dra. Isabel Guzmán Ibarra \\ Dra. Ma. Concepción Soto Valenzuela \\ Universidad Autónoma de Chihuahua, México
}

\begin{abstract}
The purpose of this article is to present results of the application of a didactic strategy to learn chemistry in higher education based on the Model for the Development and Evaluation of Academic Competences. The study was carried out with second semester students of the subject "Chemical processes of human life" of the Bachelor of Nutrition. Didactic sequences were built and applied in a classroom intervention, the structure of which includes two devices: one for training and the other for authentic evaluation; The training device uses real problem situations in the profession, the resolution of which involves the mobilization of knowledge and the development of skills. The classroom intervention process included moments of reflective practice and teacher accompaniment. The research-trainingaction approach carried out incorporates the qualitative analysis of data collected through video recording, notebooks and reflective observation of the intervention. It is concluded that the didactic strategy produces gradual changes when moving from a traditionalist teaching style to a transformed and transformative innovative educational practice.
\end{abstract}

Keywords: : didactic sequences, problem situations, chemical processes, chemistry didactics

\section{Introducción}

Para muchos estudiantes de carreras del área de ciencias de la salud el estudio de la química representa un reto, pues ello conlleva el razonamiento y análisis de conceptos teóricos de naturaleza abstracta y su comprensión requiere el desarrollo del pensamiento crítico. Esta problemática no es exclusiva de un espacio o edad de los estudiantes, pues se observa en los niveles de secundaria, bachillerato y profesional (Castillo et al., 2013; FathiAzar et al., 2013; 2013; Garritz, 2010; Menargues, 2012). Generalmente, a los 
profesores de química les es transferida la responsabilidad de la existencia de una baja percepcion social sobre la química, señalándose en cierta medida que su actuar antepone el aspecto pragmático de la ciencia dejando de lado su papel de profesores ante un grupo de estudiantes en formación, por lo que no se logran enaltecer sus alcances y trascendencia (Edwards et al., 2016; Herradón, 2012).

Para estudiantes de carreras del campo de la salud la química es una asignatura básica, resultando primordial el adquirir su conocimiento y lograr la integración a la práctica de estos conocimientos de carácter científico, a fin de obtener las competencias necesarias para su futuro desempeño profesional (Ausubel et al., 2006). Esta integración es la principal meta de la educación en ciencias y hace necesario que su enseñanza posea relevancia dentro de un contexto práctico, ya que comúnmente se enseña con base en conceptos teóricos, lo cual induce a la memorización (Lazo, 2012; Martínez, 2014).

Lo anterior, evidencia que las más diversas propuestas y tendencias educativas no han generado los cambios esperados, encontrando que las posiciones convencionales, en cuanto al campo pedagógico o bien al eclecticismo imperante, actualmente se encuentran articulados a los programas de las asignaturas y a las competencias de los estudiantes (Escribano, 2017).

Respecto del papel que juega el profesor en el proceso de aprendizaje de sus estudiantes, planteamos que este deberá ser de mediador a fin de ayudarles a superar las situaciones problemáticas que enfrentan. Asimismo, encaminarlos hacia su formación integral ante fenómenos complejos (Blanchard \& Muzás, 2016), los cuales, recurrentemente encapsulan a los estudiantes en realidades desfiguradas que se les han introyectado. Estas realidades constituyen las dificultades que entraña el aprendizaje de la ciencia, y con ella de la química, provocando aversión, miedo y apatía frente a lo que les presentan como desconocido y difícil de aprender, que, además de ser incomprensible, figura como una visión alejada de su vida cotidiana o con niveles de abstracción que parecen estar fuera de su alcance, cuando en realidad son fenómenos que forman parte de su existencia (Fernández y Moreno, 2008; Matlin et al., 2016; Reibstein, 2017).

Con base en lo descrito, en este estudio se plantea la necesidad de construir estrategias didácticas desde el enfoque de competencias que mejoren el nivel de aprovechamiento de la química en estudiantes universitarios del área de la salud. El objeto de estudio se enfoca a la atención de los procesos de enseñanza aprendizaje de la química en los estudiantes de segundo semestre de la asignatura "Procesos químicos de la vida humana", de la Licenciatura en Nutrición; dicha asignatura proporciona conocimientos para el desarrollo de las capacidades y para la toma de decisiones, además, sienta las bases de los saberes en ciencias que permiten comprender los mecanismos bioquímicos de 
la persona y su entorno. (Alvarado, 2012; Cuenca, 2011; Holbrook, 2005; Ortega, 2015).

En ese sentido, el propósito de este artículo es presentar una parte de los resultados de dicha investigación, en la cual se construyó y aplicó, en un proceso de intervención áulica, una estrategia didáctica para la enseñanzaaprendizaje de la química en la educación superior, la cual se encuentra sustentada en el Modelo para el Desarrollo y Evaluación de Competencias Académicas (Guzmán et al., 2014), M-DECA en lo sucesivo.

Se consideró que el M-DECA se adapta a las necesidades y objeto de estudio de esta investigación. Este modelo, en su estructura y operación, se basa en el diseño y puesta en práctica de secuencias didácticas, las cuales emplean una tipología de actividades agrupadas en dos dispositivos: uno de formación, basado en la pedagogía de la integración (Roegiers, 2010), la teoría de las situaciones didácticas (Brousseau, 2007) y otro de evaluación, que se sustenta en la evaluación auténtica (Brown, 2015; Cárdenas, y Pastrana, 2016; Monereo, 2009; Moria \& Zaim, 2017).

El M-DECA propone que las experiencias vividas en el proceso de enseñanza-aprendizaje, deben ser reales, situadas y sustentables para obtener el bagaje de competencias requerido y le sean significativas a los estudiantes (Guzmán et al., 2014). De tal forma, promueve el desarrollo de las competencias académicas y su evaluación mediante momentos reflexivos y de acompañamiento para el estudiante, además, señala que los elementos del concepto de competencias marcan niveles de coherencia que se trasladan e instalan en las prácticas educativas y su correspondiente evaluación, estableciendo así, una alineación constructiva que expresa y articula los componentes que integran el modelo: formación-intervención-evaluacióninvestigación. Guzmán et al (2014) definen competencia como la capacidad o aptitud de una persona para la movilización interna de recursos cognitivos para responder de forma pertinente a una situación problema.

\section{La Enseñanza de la Química: Competencias y Teoría de las Situaciones}

La teoría de las situaciones sostiene que el estudiante aprende adaptándose a entornos que le presenten dificultades y contradicciones, haciendo uso de su capacidad para aprender solucionando esas dificultades (Brousseau, 2007). Una situación proporciona momentos de aprendizaje cuando los estudiantes se enfrentan a un problema y buscan su solución utilizando sus conocimientos y apropiándose de los que faltan, ya sea individualmente o con sus pares sin la intervención del docente (Brousseau, 2007; García y Posso, 2017; Panizza, 2003), analizando, modificando o construyendo sus conocimientos a través de una sucesión de actividades que les permiten desarrollar competencias situadas dentro de un contexto definido a través del diálogo y la reflexión (Guzmán et al., 2014). Se considera que: 
Las competencias no pueden definirse sino en función de situaciones, están tan situadas como los conocimientos en el contexto social y físico. El concepto de situación se vuelve el elemento central del aprendizaje: es en situación que el alumno se construye, modifica o refuta los conocimientos contextualizados y desarrolla competencias a la vez situadas. [...] Ya no basta con enseñar contenidos disciplinares descontextualizados (área del trapecio, suma de fracciones, procedimiento de cálculo mental, reglas de sintaxis, modo de conjugación, etc.) sino de definir situaciones en las cuales los alumnos pueden construir, modificar o refutar conocimientos y competencias a propósito de contenidos disciplinares (Jonnaert et al., 2008, p.3).

Al trabajar por competencias, el concepto de situación no solo se vuelve indiscutible sino además insustituible. Si una situación representa para el estudiante un obstáculo o un reto a vencer, y cuya solución genera nuevos aprendizajes, entonces la llamamos "situación problema"; en ese sentido, "La situación-problema y el objetivo obstáculo regulan, potencian, contribuyen en el aprendizaje en el estudiante." (Sarzosa y Pérez, 2017, p.596). Bajo la noción de "contrato didáctico" la retroalimentación de las situaciones puede darse en ambientes caracterizados por ser eminentemente didácticos o pueden estar referidos a medios a-didácticos; con esta última noción se acude a un "nuevo contrato didáctico" y, con ello, al incumplimiento del contrato convencional (implícito, no escrito), en donde, el rol de enseñar está depositado en la figura del profesor y el de aprender recae en el estudiante.

La naturaleza del aprendizaje de ciencias, conlleva buscar conocimientos significativos desde el inicio, esto implica que continuamente el estudiante mantenga su estructura mental preparada para la recepción de nuevos conocimientos cada vez más desarrollados y complejos, pues los primeros sirven de ancla para los siguientes, tal como sucede en los aprendizajes de la química que se construyen en un continuo por modificación de ideas, razonamiento y el marco de referencia personal (Ausubel et al., 2006; Bretz et al., 2013; Díaz-Barriga y Hernández, 2010; Lazo, 2012).

Asimismo, se considera importante abordar el conocimiento científico desde perspectivas socioconstructivistas capaces de llevar al estudiante a generar y potencializar el aprendizaje de la química, considerando sus bases conceptuales para comprenderla y mediante la construcción y puesta en práctica de situaciones didácticas que acrecienten su formación en en cuanto a dotarlos de mayor autonomía, y hacerlos más conscientes y sociocríticos (Sarzosa y Pérez, 2017). Adoptar una postura sociocrítica conduce a los estudiantes al análisis minucioso y en contexto "de la situación o problemática de la realidad social a entender, que promueva la idea de vivir juntos, en favor 
de la realización de cambios necesarios para la superación de las problemáticas existentes." (Grijalba et al., 2020, p.67)

El M-DECA propone el aprendizaje por competencias a partir de situaciones problema que permiten la reflexión y la interiorización de saberes, cumpliendo también con las dos condiciones para alcanzar la significancia: a) que los temas sean interesantes, y b) que permanezcan; es por ello que las secuencias didácticas se estructuran de manera que favorecen la disposición para apropiarse del conocimiento al resolver un problema.

Dadas sus características, el M-DECA puede ser utilizado como andamio cognitivo para estudios o investigaciones en educación y adquisición de competencias, por lo que se considera apropiado para la didáctica de la química (Guzmán et al., 2014; Marín et al., 2015). Dos características que lo hacen pertinente es que considera los dos aspectos fundamentales en el aprendizaje: la formación y la evaluación, y en ambas, los estudiantes participan activamente. Además, consta de los cuatro componentes teóricometodológicos mencionados que lo hacen integral: conceptual, formación, evaluación, e investigación.

El componente conceptual, como se señaló antes, define la competencia como un conjunto de saberes y actitudes para tomar acción ante una situación y los recursos para afrontarla. Este conjunto es la base de las estrategias de formación y evaluación; el dispositivo de formación, se enfoca en lograr las competencias de la asignatura, razón por la cual las secuencias parten de situaciones encaminadas al análisis e interiorización de saberes a través de actividades de aprendizaje. Las situaciones problema deben ser complejas y abiertas, lo cual mejora la capacidad del pensamiento crítico, las capacidades cognitivas básicas, el razonamiento y, por ende, el aprendizaje de la química. (Ausubel, 2006; Del Valle, 2008; Díaz-Barriga, 2006; Roegiers, 2010)

El componente de formación se estructura mediante una tipología que posee cuatro elementos: a) situación problema, b) actividades de aprendizaje, c) evidencias de desempeño, y d) recursos de apoyo.

El componente de evaluación, influye en la disposición para elaborar constructos y, con ello, obtener aprendizajes (Roegiers, 2016). La interacción de los actores; profesor, estudiante y materiales o actividades educativas apoyan el logro de los aprendizajes necesarios y las competencias para el análisis de información, comprensión científica y rigor analítico, desarrollando también el enfoque lógico, pragmático, analítico y argumentativo (Moreira, 2009, 2012; Overton, 2013; Sarzosa y Pérez, 2017; Thron, 2010).

El componente de investigación, evalúa la intervención áulica al validar el modelo y evaluar las acciones formativas, tal como se efectúa en una investigación-acción-participativa (Elliott, 2005). Se propone para 
realizarse en tres etapas: 1) Cosituacional, valora el enfoque, aprendizajes y nivel de construcción, 2) Cooperacional, el profesor-investigador realiza la intervención en el aula e investiga la experiencia, y 3) Coproducción, análisis de la experiencia en grupo (Guzmán et al., 2014).

\section{Método}

Este estudio se plantea como un trabajo con enfoque cualitativo. Un aspecto medular en esta investigación se centra en un programa de formación de profesores, donde se construyen secuencias didácticas basadas en situaciones problema, las cuales se implementan en una intervención en el aula. Esto significa, no solo el dominio de saberes disciplinares, sino además, implica incursionar en los campos pedagógico y didáctico en lo referente al diseño de los ambientes de aprendizaje para el desarrollo de competencias, asimismo, en el análisis permanente de la forma en cómo se establecen las interacciones en las aulas para el logro de competencias estudiantiles; esto es, transitar del plano de los saberes pedagógicos al espacio de los saberes didácticos (Zambrano, 2019).

Nuestro estudio, puede considerarse como una investigaciónformación-acción en la cual se recorren momentos metodológicos fundamentales en donde se analizan "la pregunta por la naturaleza del saber enseñado, la relación sujeto-objeto, la producción de conceptos didácticos, la relación con el saber en la doble perspectiva sujeto-objeto-sujeto, la génesis del saber enseñado, las representaciones del saber, entre otros." (Zambrano, 2019, p.80).

Se estimó conveniente adoptar como método la investigaciónformación-acción docente, entendida como aquella en la cual los participantes "tengan oportunidad de relatar, junto con otros, historias acerca de su práctica docente y para que esas formas de interpretación del mundo escolar sean puestas en escritura, indagación, deliberación y cambio." (Suárez y Metzdorff, 2018, p. 50).

Mediante la participación en procesos reflexivos y creativos los profesores-investigadores participan en este doble rol, como sujetos y objetos de su propio proceso de formación e intervención áulica (Soto, 2015). La investigación-formación-acción surge de los cuestionamientos a los procesos de formación e intervención áulica basados en una racionalidad técnicoinstrumental actual, con el propósito de incursionar en una racionalidad crítico emancipadora (Habermas, 1986).

La investigación-formación-acción fundamenta sus principios en perspectivas metodológicas que emanan de la postura (auto)biográficanarrativa (Bolívar y Domingo, 2019; Huchim y Reyes, 2013), de la investigación (auto)etnográfica (Alegre-Agís y Riccò, 2017; Blanco, 2012) y de la investigación-acción en el salón de clases (Anderson y Herr, 2007; 
Batallán, 2007; Eliott, 2005); donde adopta particularidades de investigaciónacción participativa en el aula; en esta última, los docentes adoptan el rol de profesores-investigadores que participan en el desarrollo del currículo desde una posición de generación de conocimiento sobre y para la docencia.

Desde esta postura de investigación-formación-acción, se realiza una observación reflexiva de cómo se produce el conocimiento en el aula, ya sea procesos de aprendizaje cooperativo, de rigor científico o de carácter analítico, así como de las competencias transversales, como el saber actuar, primordial en el desempeño profesional (Marín et al., 2015). Las observaciones realizadas fueron de tipo participativo, los estudiantes agrupados en tríadas realizaron trabajo colaborativo e interacción grupal (Medrano et al., 2015).

\section{Muestra}

El estudio se desarrolló en la Facultad de Enfermería y Nutriología de la Universidad Autónoma de Chihuahua (UACH). La muestra fue el grupo de estudiantes $2 \ln 2$ de segundo semestre de la materia de "Procesos químicos de la vida humana", compuesto por 21 estudiantes (11 de ellos son mujeres), con edades de 18 y 21 años de edad. Del total de estudiantes, 10 se inscribieron a la materia por primera vez, siete por segunda y cuatro por tercera ocasión. La asignatura se cursó en 48 horas teóricas y 32 horas en laboratorio; se realizó en turno vespertino con tres horas los días martes y dos horas los viernes. En la integración del grupo no hubo criterios de inclusión o de exclusión.

\section{Procedimiento}

El procedimiento metodológico, contempló cuatro etapas:

Primera etapa: formación. Se realizó un diplomado que incluyó el aprendizaje de los fundamentos del M-DECA, fue empleado como base de esta investigación para observar la adquisición de las competencias en cuestión: formación continua, diseño de la docencia, transposición didáctica, progresión de las competencias, comunicación educativa, y evaluación del logro de las competencias (Guzmán et al., 2014).

Segunda etapa: construcción y diseño de secuencias didácticas. Las secuencias didácticas se construyeron enfocándolas a los objetos de estudio de la asignatura de "Procesos químicos de la vida humana". Se diseñaron 11 secuencias didácticas, como guías para el estudiante en el proceso de intervención, que agrupan los contenidos (naturaleza química del ser humano, carbohidratos, lípidos, proteínas, enzimas, ácidos nucleicos, vitaminas y minerales, agua, electrolitos y buffers, sangre y respiración, y acción hormonal) del programa de la asignatura. Las secuencias construidas individualmente fueron inicialmente valoradas al interior de cada tríada de profesores mediante una rúbrica. Se procedió a implementar los ajustes y las adecuaciones que se indicaron $\mathrm{y}$, en un segundo momento, las secuencias 
fueron evaluadas por un grupo de expertos que las validó, generando algunas sugerencias de correcciones sobre aspectos de contenidos y refinamiento de los instrumentos, las cuales se aplicaron, en un tercer momento, fueron presentadas ante el grupo para su validación final, quedando listas para su utilización (Guzmán et al., 2014).

Las situaciones problema que se diseñaron fueron reales, retadoras y motivadoras, para ello, se consideraron tópicos actuales y relevantes sobre cada tema y se incluyeron preguntas generadoras de mayores niveles de participación, colaboración y dominio. Con esto se buscó que los estudiantes percibieran la relación directa existente entre la escuela y su futuro quehacer. Asimismo, que lograran aprendizajes mientras realizaban trabajo reflexivo, integrando los saberes previos con los nuevos.

Del total de secuencias diseñadas se presentan en enseguida las situaciones problema de las secuencias dos y ocho (carbohidratos y agua), de su aplicación en la intervención áulica se describen los resultados en el apartado correspondiente.

\begin{tabular}{|c|c|c|}
\hline Unidad de análisis & Categoría de análisis & Situación problema \\
\hline Secuencia didáctica 2 & Situación problema & Carbohidratos \\
\hline
\end{tabular}

Los carbohidratos son los compuestos orgánicos más abundantes en la naturaleza, como ejemplo la glucosa la principal fuente de energía y la molécula central del metabolismo general de todos los seres vivos aerobios. Dada su importancia adquiere relevancia el conocerlos y poder comprender cómo contribuyen con el metabolismo del ser humano, ante las diferentes situaciones en la alimentación, el ayuno, el ejercicio, etc., cuál es su papel en una de las enfermedades más comunes en nuestro país: la diabetes, patología a la que un Nutriólogo se enfrentará continuamente en su quehacer profesional. Siendo ésta la parte medular de esta situación-problema, su solución da razón para el planteamiento de los siguientes cuestionamientos:

1. ¿Qué relación guarda la glucosa con la insulina y el glucagón en la diabetes?

2. ¿Cuáles son las funciones de las Glucoproteínas y cuál es su utilidad en la respuesta inmune?

3. ¿Qué utilidad tendrá conocer sobre los Carbohidratos en mi desempeño profesional?

\begin{tabular}{|c|c|c|}
\hline Unidad de análisis & Categoría de análisis & Situación problema \\
\hline Secuencia didáctica 8 & Situación problema & Agua \\
\hline
\end{tabular}

El agua líquida formada por Oxígeno e Hidrógeno es el componente más importante y abundante sobre la superficie terrestre, sin ella la vida no hubiera sido posible ya que sus características y propiedades fisicoquímicas hacen posible que muchos organismos se desarrollen en los océanos, pero también en ambientes adversos como los desiertos donde hay poca agua o donde el agua es caliente o muy fría como en los polos.

Tomando en consideración que el agua es vital para el desarrollo general del metabolismo de los seres vivos y está presente en cualquier proceso normal o anormal, se toman para su estudio estos cuestionamientos: 
1. Se ha descrito que el agua es el disolvente universal, Si esta declaración fuera estricta. ¿Podría haber surgido la vida sobre la tierra? ¿Cómo las propiedades del agua intervienen?

2. Muchas moléculas son polares, aunque no formen enlaces de hidrógeno significativos ¿Que tiene tan poco usual el agua que hace posible el enlace de hidrógeno? ¿Cómo se relaciona el agua con otras biomoléculas?

3. El hielo es menos denso que el agua. Si no fuera así ¿Cómo se verían afectados los océanos? ¿Cómo se vería afectado el desarrollo de la vida en la Tierra?

4. ¿De qué manera participan los puentes de Hidrógeno en la transferencia de información genética?

5. El estudio del agua y sus propiedades bioquímicas tiene una utilidad y aplicación en el ámbito de mi carrera profesional ¿cómo sustento esta afirmación?”

Tercera etapa: intervención áulica con el grupo de estudiantes. El proceso de intervención se desarrolló a partir de las secuencias didácticas diseñadas y validadas, aplicadas al grupo-muestra. La aplicación de las secuencias se dio en un proceso de intervención áulica, el cual se observó y registró mediante: 1) La videograbación de los acontecimientos durante el desarrollo de cada sesión de clase; el tiempo total de las videograbaciones en el aula y en el laboratorio fue de 30 horas, en ellas se incorpora el trabajo colaborativo y la interacción de las tríadas y del grupo, incluyendo prácticas de laboratorio donde se recrean los conceptos teóricos de las secuencias didácticas de manera experimental, 2) Una bitácora que contiene la descripción escrita de los hallazgos relevantes acontecidos durante la intervención, y 3) Registro de la observación detallada y reflexiva del desarrollo de la intervención.

Cuarta etapa: valoración de la experiencia. Los resultados obtenidos se analizaron buscando comprender cómo fue la experiencia para los estudiantes. Asimismo, se realiza la descripción holística de la investigación puntualizando cómo los estudiantes interactuaron. El análisis de los hallazgos proporcionó información sobre el grado de conocimiento desarrollado y permitió comprender sus significados.

\section{Análisis de los datos}

La sistematización de los datos se realizó en dos grandes momentos.

1) Organización de la información: esta se dio a partir de la Matriz de Análisis de la Práctica Educativa (MAPE). Se tomó como Unidad de Análisis a las secuencias didácticas, como Categorías de Análisis a los elementos que las conforman (intenciones formativas, situaciones problema, actividades de aprendizaje y evidencias de desempeño), y como Criterios fueron considerados, entre otros: univocidad, apropiación, realidad, significatividad, pertinencia, relevancia, integración, y desempeños. Esta organización facilitó los procesos de reducción de datos, su clasificación y categorización logrando establecer relaciones entre lo planeado y lo logrado en las diferentes categorías 
y criterios trabajados: intenciones formativas situaciones problema, evidencias de desempeño, actividades de aprendizaje, recursos de apoyo y evaluación, bajo esta lupa, el análisis resultó enriquecedor.

2) Análisis e interpretación: a partir de los datos organizados, en este momento se profundizó en el análisis e interpretación mediante la teoría fundamentada, la cual permitió arribar a productos y explicaciones o conceptualizaciones teóricas "con un marco explicativo para entender el fenómeno estudiado, en un proceso ordenado y constante de comparación, análisis y codificación.” (De la Espriella y Gómez, 2020, p.127). La teoría fundamentada se manejó en tres niveles de análisis, donde la codificación fue básica, implicando una lectura detallada y exhaustiva para la identificación de subtemas. La codificación realizada fue de tres tipos: abierta, esta permitió encontrar categorías en los datos; axial, con ella se hallaron relaciones entre categorías; y selectiva, donde se pretendió explicar las relaciones y localizar las categorías centrales. (De la Espriella y Gómez, 2020) este análisis se apoyó en el programa Atlas Ti 7.5.7 (San Martín, 2014).

\section{Resultados}

En este artículo se presentan resultados parciales de la investigación, estos corresponden a la valoración de la tercera etapa de la investigación referida al proceso de intervención en el aula. En el análisis general de estos resultados para su presentación se tomaron, como representativas para el análisis a profundidad, a cinco de las 11 secuencias trabajadas con los estudiantes $(2,8,9,10$ y 11). Aquí se presentan los resultados de las «Categorías»: situación problema, actividades de aprendizaje, y evidencias de desempeño, de las secuencias dos y ocho (carbohidratos y agua), descritas en la etapa dos de la sección de procedimiento metodológico. Los resultados se ofrecen en matrices construidas a partir de los testimonios o episodios verbales y su interrelación con los «criterios de análisis», definidos en el MAPE.

\section{Situaciones problema}

Dada la importancia y trascendencia en el estudio, en esta sección se presentan los resultados de la aplicación de dos situaciónes problema: primero, la del tema de carbohidratos, y enseguida, la del agua (ver su estructura en etapa dos del procedimiento metodológico).

En la situación problema de la secuencia del tema carbohidratos se transversalizó un tema muy importante: la diabetes - segunda causa de muerte en México-, y uno de los principales motivos de atención nutriológica. Esto sitúa a la situación en un contex to relevante en el quehacer del nutriólogo; así, se consideró pertinente su inclusión en la situación, orientando su última pregunta a una reflexión crítica. En el aula se dió lo siguiente: 


\begin{tabular}{|c|c|c|}
\hline Estudiante & Episodio verbal & Comentario \\
\hline $21 \mathrm{fP}(\mathrm{T} 1)$ & $\begin{array}{l}\text { Los monosacáridos son } \\
\text { azúcares tales como el azúcar } \\
\text { de mesa o azúcar de la fruta } \\
\text { que es la fructosa, maíz- } \\
\text { dextrosa y glucosa. }\end{array}$ & $\begin{array}{l}\text { La actividad consistía en explicar las } \\
\text { características Fisicoquímicas de los } \\
\text { monosacáridos. Habló leyendo de su } \\
\text { cuaderno en voz alta. Se presentó la } \\
\text { lectura de una oración, la cual no } \\
\text { contenía la información solicitada, } \\
\text { nombrando compuestos que no eran } \\
\text { monosacáridos y que no se } \\
\text { referenciaban o comparaban. }\end{array}$ \\
\hline Docente & $\begin{array}{l}\text { ¿La sacarosa es mono o } \\
\text { disacárido? }\end{array}$ & $\begin{array}{l}\text { Pregunta de apoyo docente. La respuesta } \\
\text { correcta era: el azúcar de mesa es la } \\
\text { sacarosa y es un disacárido, y la dextrosa } \\
\text { es un oligosacárido. Se denotaba } \\
\text { confusión en los términos y no se } \\
\text { advertía estudio previo, solo ideas vagas } \\
\text { y algunas erróneas. }\end{array}$ \\
\hline $20 \mathrm{Fp}(\mathrm{T} 1)$ & $\begin{array}{l}\text { Maestra, pero en } 10 \text { min no } \\
\text { vamos a poder aprender todo. }\end{array}$ & $\begin{array}{l}\text { Interviene otra integrante de la tétrada. } \\
\text { Los } 10 \text { minutos se otorgaron para que } \\
\text { organizaran los materiales de acuerdo a } \\
\text { los roles de cada integrante. }\end{array}$ \\
\hline Docente & $\begin{array}{l}\text { Pero en las actividades previas } \\
\text { Uds. estudiaron el tema y } \\
\text { sabian el subtema a } \\
\text { desarrollar. }\end{array}$ & Silencio... \\
\hline Docente & $\begin{array}{l}\text { ¿Cuántos } \quad \begin{array}{r}\text { monómeros } \\
\text { contienen } \\
\text { simples? }\end{array} \\
\end{array}$ & $\begin{array}{l}\text { Ayuda docente, buscando crear diálogo } \\
\text { y alguna pregunta introductoria y } \\
\text { sencilla a la vez para romper el silencio. }\end{array}$ \\
\hline $20 \mathrm{fP}$ & $\begin{array}{l}\text { Son de } 3 \text { a } 7 \text { átomos de } \\
\text { carbono. }\end{array}$ & $\begin{array}{l}\text { Su respuesta era correcta, ya que } \\
\text { respondió sobre átomos de carbono y no } \\
\text { monómeros de monosacárido. }\end{array}$ \\
\hline Docente & ¿La sacarosa de cuantos es? & Silencio prolongado. \\
\hline $21 \mathrm{fP}$ & $\begin{array}{l}\text { Los monosacáridos son } \\
\text { azucares, tales como el azúcar } \\
\text { de mesa que es una azúcar } \\
\text { simple y es de } 3 \text { a } 7 \text { átomos de } \\
\text { carbono. }\end{array}$ & $\begin{array}{l}\text { Toma la palabra, repite parte de lo ya } \\
\text { expresado y que era incorrecto. } \\
\text { Confusión entre átomos de carbono y } \\
\text { moléculas de monosacáridos, } \\
\text { disacáridos y oligosacáridos. }\end{array}$ \\
\hline Docente & $\begin{array}{l}\text { Cuando nos referimos a } \text { un } \\
\text { disacárido no es de } 3 \text { a } 7 \\
\text { átomos de carbono sino } 12 \ldots .\end{array}$ & Ayuda docente aclarando los términos. \\
\hline
\end{tabular}

Se escogió esta secuencia para ilustrar a cómo se desarrollaron las primeras sesiones de clase, con cambio de roles de docente y estudiantes, esto es, un nuevo «contrato didáctico»-implícito- (Brosseau, 2007).

En las primeras sesiones se realizaron ajustes y mejoras a las secuencias y a sus actividades de aprendizaje, motivados por la resistencia al cambio, por parte de los estudiantes, ante una relación educativa que les 
implicaba mayor esfuerzo al aprender a través de una nueva metodología. Inicialmente, se presentó, en forma de secuencias, el nuevo contrato didáctico, pero no se "negoció", solo se asumió como una nueva propuesta de trabajo.

Conforme se hizo evidente el compromiso de desarrollar, dentro de las actividades de aprendizaje, lecturas previas y entregables en tiempos establecidos, esto provocó conflicto manifiesto en segmentos de interactividad tensos, esto puso en riesgo la relación estudiantes-docente; llevando a momentos de malestar, como se observa en la matriz anterior.

Esta secuencia de Carbohidratos, establece lecturas previas del tema a fin de refrescar lo conceptual tratado en el semestre anterior. Se recomendó la lectura del capítulo de carbohidratos en dos libros de bioquímica, ambos adecuados al nivel de estudiantes de segundo semestre de licenciatura. En el diseño de las actividades de aprendizaje se indicaba que en la clase previa, se analizara un video alusivo al tema, además, que el docente estableciera un diagnóstico respecto del nivel de conocimientos del grupo.

De acuerdo con los resultados se estimó necesario realizar un repaso conceptual para aterrizar conceptos. De esta forma, se acordó realizarlo durante la siguiente clase, antes de entrar a las actividades para abordar la situación problema. Para esta actividad se distribuyó material impreso para su análisis en las tríadas.

Este cambio en la progresión de las secuencia fue uno de los primeros en los que se observó una distancia entre lo planeado y lo realizado, esto generó negociaciones para cambios en la docencia.

A partir de este acuerdo, los estudiantes realizarían las actividades de aprendizaje como estaban definidas en las secuencias, pero a cambio, la demanda de los estudiantes se centró en que al final de la clase el docente diera una "exposición magistral" sobre el tema abordado. Ante esto, se incluyeron ajustes en la organización grupal, patrones de integración para el trabajo didáctico y el orden de algunas actividades de aprendizaje.

Se accedió a la solicitud de los estudiantes buscando no crear mayor controversia, estimando que el proyecto podría encontrase en riesgo. El nuevo contrato didáctico, tenía dos aspectos a cuidar: el compromiso de los estudiantes de asumir su nuevo rol que implicaba el aprendizaje autónomo con la ayuda del profesor y, la preocupación de la docente por cuidar esta regla y realizar un rol de acompañamiento y apoyo (Brousseau, 2007; Guzmán et al., 2014).

Los ajustes se realizaron a partir de la siguiente secuencia, donde se desarrolló una curva de aprendizaje "transicional" hacia el establecimiento de una nueva metodología, de un ambiente de aprendizaje distinto y de nuevas relaciones grupales. En esta transición se logró captar el interés del grupo y crear conciencia de que estaban logrando aprendizajes de los temas a través del diálogo en tríadas y grupalmente. Esta modificación se tomó dado que ésta 
era la segunda secuencia y los estudiantes apenas estaban conociendo la nueva metodología y se advertían desorientados ante las cláusulas - no escritasque establecía el nuevo contrato didáctico.

En la secuencia 8 se aborda la situación problema del tema del Agua (ver su diseño y estructura en la etapa dos de la sección de procedimiento metodológico). Se resalta que la estructura de la situación problema fue determinante para su desarrollo en el aula, siendo el detonante del principal conflicto cognitivo a nivel grupal que logró captar el interés de los estudiantes, haciendo evidente, para ellos, la distancia entre los saberes que poseían y los que ignoraban.

Con base en los acuerdos establecidos en la secuencia dos, relacionados con el apoyo del docente en la exposición del tema al final de la sesión que sumarizaba la secuencia abordada, y en el análisis conceptual individual de los estudiantes, previo a la situación problema; durante la clase se efectuaría el análisis de la situación problema a nivel de las tríadas, seguido por un debate grupal y al cierre la explicación docente sobre el tema.

Se observó que la mayoría de los estudiantes habían llevado a cabo las lecturas previas, quienes no, realizaban las dos actividades de manera simultánea. Las tríadas que iniciaron el debate presentaron una solución al problema, que parecía lógica, fundada en el conocimiento de conceptos poco profundos pero enfocados de manera incorrecta, el conflicto cognitivo se dio porque ellos consideraban que sus apreciaciones sí eran correctas, pero no lograban arribar a la solución. La situación problema favoreció este momento de coyuntura y desconcierto grupal, donde el apoyo docente y la rápida transposición didáctica fueron determinantes para que los estudiantes llegaran a la solución.

\begin{tabular}{|c|l|l|}
\hline $\begin{array}{c}\text { En sus } \\
\text { tríadas }\end{array}$ & \multicolumn{1}{|c|}{ Testimonio } & \multicolumn{1}{|c|}{ Comentario } \\
\hline $\begin{array}{c}\text { Varios } \\
\text { estudiantes }\end{array}$ & $\begin{array}{l}\text { ¿Cuáles moléculas no se pueden } \\
\text { disolver en el agua? }\end{array}$ & $\begin{array}{l}\text { Varios estudiantes se preguntan } \\
\text { entre ellos en voz baja. }\end{array}$ \\
\hline $03 \mathrm{mP}$ & $\begin{array}{l}\text { No sé exactamente como, pero pues } \\
\text { las moléculas de los lípidos no se } \\
\text { pueden disolver en el agua, como que } \\
\text { ejemplo, pero las moléculas en la } \\
\text { célula no se pueden disolver. }\end{array}$ & $\begin{array}{l}\text { Menciona dudando y gradualmente } \\
\text { toma confianza y trata de explicarlo } \\
\text { que no alcanza a concretar } \\
\text { totalmente pero su razonamiento si } \\
\text { es correcto. }\end{array}$ \\
\hline $05 \mathrm{mP}$ & $\begin{array}{l}\text { Las excepciones podrían ser las } \\
\text { hidrofóbicas ese tipo de moléculas no } \\
\text { se pueden disolver en el agua. }\end{array}$ & Reafirma sus concepciones \\
\hline
\end{tabular}

Los estudiantes avanzados del grupo lograron argumentar la solución correcta, abriendo la pauta a todo el grupo, entendiendo que las soluciones estaban a su alcance. Esto dio la confianza que necesitaba el grupo, los menos 
avanzados, confiaron en que lo podían lograr. Con esta secuencia se dió un cambio cognitivo tal que les permitió arribar a conclusiones.

Las situaciones problema fueron percibidas por los estudiantes como significativas, pertinentes y enfocadas, lo cual sucede cuando hacen conciencia que esos saberes son de aplicación o similares con los entonos profesionales que corresponden a su carrera. Otro aspecto relevante fue que pudieron hacer conciencia de su capacidad para aprender por sí mismos, al apropiarse de saberes e integrarlos a contextos reales, esto se evidenció al analizar las interrelaciones entre las diferentes categorías que emergieron del análisis con Atlas Ti, siendo las principales: apropiación e integración de saberes, significatividad y realidad y contexto, tal como se corroboró en el análisis de sus comentarios los cuales se observan en la Figura 1.

Figura 1. Percepción y categorización de las situaciones problema

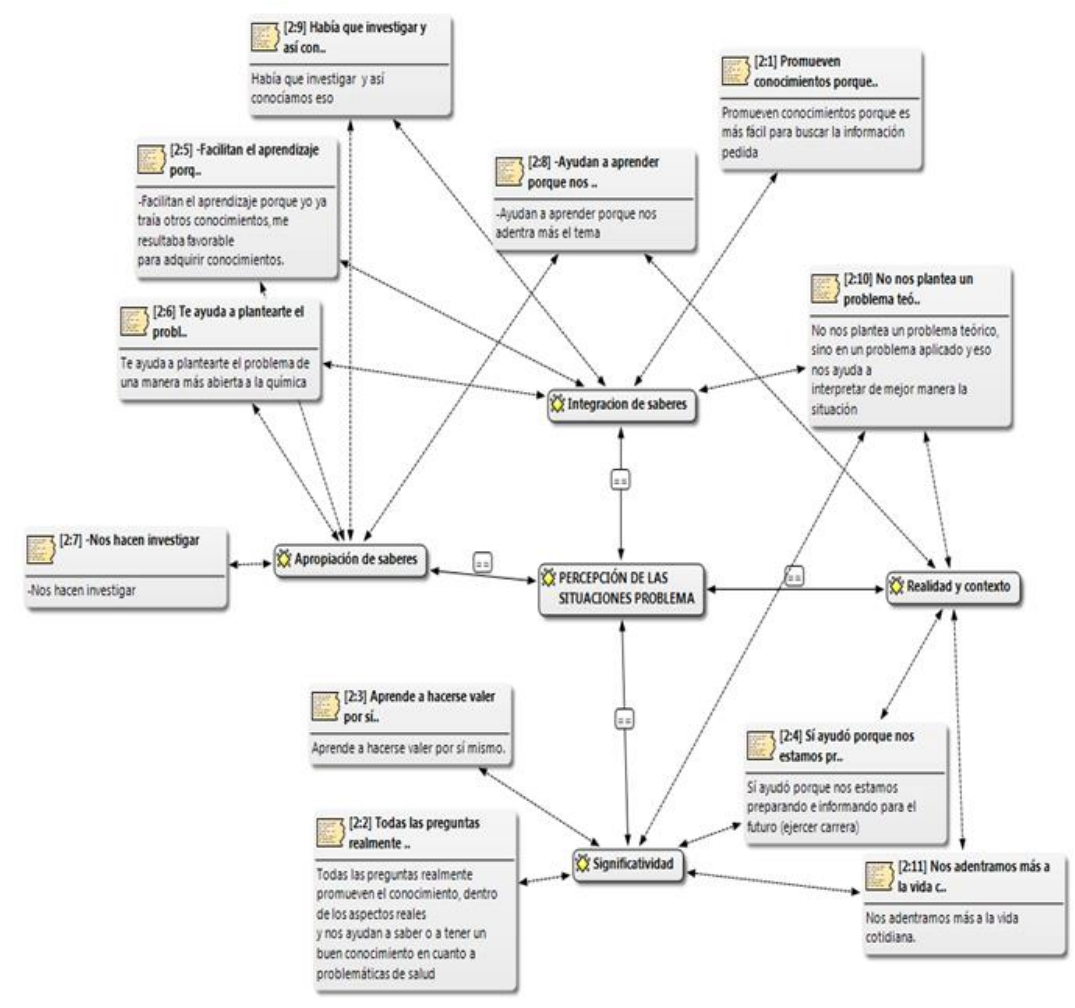

\section{Actividades de aprendizaje}

En esta categoría, durante la intervención en el aula con la secuencia ocho (tema Agua), se observó una evolución hacia la nueva metodología de aprendizaje. Los estudiantes realizaron las actividades previas propuestas, se incorporaron al tema con mayor facilidad y sus diálogos fueron más reflexivos y críticos. 
Las vivencias que se dieron en esta secuencia representan un parteaguas, marcan el momento de transición entre el antes y el después, el cambio de paradigma en el cual los estudiantes tomaron conciencia de lo que significaba la situación problema y de que podían lograr su solución.

Quienes aún no habían iniciado el proceso de realizar actividades previas, tocaron fondo, se enfrentaron a un conflicto, haciendo evidente que no es posible solucionar un problema sin poseer conocimientos básicos.

Al final de esta secuencia se efectuó la acordada explicación magistral sobre el tema trabajado. Sin embargo, en esta secuencia adquirió otra dimensión, pues los estudiantes advirtieron que, siendo los mismos conceptos estudiados y comprendidos al solucionar el problema, resultaba repetitiva la explicación sobre un tema ya tratado; así, los estudiantes gradualmente dejaron de tener interés en una "cátedra" docente. Se observó cómo se desarrolló un proceso de innovación, primero en unos estudiantes, luego otros, de acuerdo a sus capacidades, conocimientos previos y formas de aprender. Fueron el acercamiento a los conocimientos, y la adquisición de seguridad y confianza, los disparadores de los logros posteriores, acorde con Perrenoud (2011, p.124), quien menciona: "a veces es necesario llegar varias veces hasta el umbral de una puerta antes de atreverse a entrar".

En las siguientes secuencias, tres de las seis tríadas del grupo, adoptaron el hábito de la lectura, análisis y diálogo colectivo del tema en estudio de manera previa a la clase. Enseguida dos tríadas se sumaron a este proceso; la tríada restante trabajó por separado.

La acción de cierre de las actividades de aprendizaje - exposición magistral del tema-, solo se realizó en las siguientes dos secuencias, pues conforme los estudiantes fueron realizando las actividades previas, aumentó su grado de análisis y comprensión, de tal forma que al final de cada secuencia ya no buscaban la seguridad de la exposición magistral, esta se substituyó por una mayor retroalimentación sobre ejemplos en contexto o aplicaciones prácticas de los temas como se observa a continuación:

\begin{tabular}{|l|l|}
\hline Estudiante & \multicolumn{1}{|c|}{ Episodio verbal } \\
\hline $12 \mathrm{mP}$ & $\begin{array}{l}\text { Hacer la clase como más de ejemplo, explicando lo químico, pero también } \\
\text { ejemplos de lo cotidiano }\end{array}$ \\
\hline $05 \mathrm{mP}$ & $\begin{array}{l}\text { Ejemplos que nos lleven a tener una vista más general de lo que nos podemos } \\
\text { topar. }\end{array}$ \\
\hline
\end{tabular}

\section{Evidencias de desempeño}

Esta categoría de la secuencia ocho describe "La reflexión y análisis de las propiedades fisicoquímicas del agua: disolventes, coligativas y térmicas, estableciendo las relaciones y diferencias entre ellas; de forma que proporcionen los elementos de análisis y solución a los cuestionamientos de la situación problema". Su diseño incluyó una reflexión con detonadores de la 
discusión, por ejemplo: el agua es el disolvente universal, si esa declaración fuera estricta ¿pudo haberse formado la vida en la tierra?

Esto define al agua como el disolvente universal y nos coloca ante un concepto trivial, pero ubica al agua como una molécula que disuelve todo, ¿cómo lo hace?, ¿realmente lo hace?, ¿por qué se ha ganado este adjetivo? Estas preguntas tienen su base en las propiedades fisicoquímicas del agua y son retomadas para explicar su comportamiento en un contexto biológico.

Esto generó la reflexión sobre teorías y conceptos, implicando su comprensión al no disponer de conocimientos previos.

Las evidencias de desempeño en esta secuencia incluyen la reflexión y análisis de los aspectos conceptuales básicos, para enfrentar las situaciones problema desde diferentes ópticas, primero de manera individual, considerando conocimientos previos, enseguida, su desempeño en el trabajo de las tríadas, posteriormente, en el trabajo grupal; en esta etapa se dio el pensamiento analítico y crítico, llegando a soluciones colaborativamente.

La siguiente matriz evidencia cómo el cuestionamiento favoreció aprendizajes del pensamiento crítico durante la intervención en el aula.

\begin{tabular}{|c|c|c|}
\hline Participante & Episodio verbal & Comentario \\
\hline $05 \mathrm{mP}$ & $\begin{array}{l}\text { Maestra, bueno yo entendí, yo } \\
\text { este.... yo diría que no se pudo haber } \\
\text { surgido la vida porque este... el } \\
\text { agua es el disolvente universal, si } \\
\text { esta declaración fuera estricta o sea } \\
\text { yo a lo que entendí, es que si } \\
\text { realmente el agua todo lo puede } \\
\text { disolver absolutamente todo jaja i... } \\
\text { No hubiera podido haber dado la } \\
\text { vida. }\end{array}$ & $\begin{array}{l}\text { Esta respuesta evidenció como la } \\
\text { situación problema fue el detonante } \\
\text { de análisis introspectivo que } \\
\text { permitió evidenciar que la } \\
\text { adquisición de saberes y el } \\
\text { pensamiento crítico se habían } \\
\text { desarrollado. El estudiante al inicio } \\
\text { dudoso, proporcionó una respuesta } \\
\text { lógica y correcta. }\end{array}$ \\
\hline Docente & $\begin{array}{l}\text { ¡Así es! ¡Es correcto!, si fuera } \\
\text { totalmente estricta, todo lo que hay } \\
\text { en la naturaleza se podría } \\
\text { disolver... ¿Se podría haber dado la } \\
\text { vida? }\end{array}$ & $\begin{array}{l}\text { Ayuda docente, motivando el } \\
\text { análisis del cuestionamiento. }\end{array}$ \\
\hline $05 \mathrm{mP}$ & $\begin{array}{l}\text { No, porque, porque no hubiera } \\
\text { podido darse la vida. Porque si se } \\
\text { hubiera formado algo, bueno si } \\
\text { pudiera haberse formado algo, } \\
\text { porque el agua lo hubiera disuelto. }\end{array}$ & $\begin{array}{l}\text { Interviene de nuevo el estudiante y } \\
\text { explica al grupo las bases teóricas } \\
\text { de su respuesta. }\end{array}$ \\
\hline
\end{tabular}

De manera adicional, evidencia el desarrollo de competencias transversales como la confianza en sí mismo, no solo en lo individual, sino que también a nivel de grupo los estudiantes desarrollaron competencias.

\section{Discusión}


Un hallazgo importante es cómo los estudiantes transitan en una sucesión de estadíos que inician en la zona de confort que brinda la metodología enseñanza-aprendizaje tradicional - tipo bancario- donde el profesor entrega conocimientos y ellos los reciben (Ordaz, y Britt, 2018). Ante la perspectiva de evaluación auténtica, basado en evidencias y, contraria a la evaluación tradicional, el proyecto fue bien acogido.

La necesidad de realizar actividades previas, de socializar las situaciones problema y de buscar soluciones con enfoques bioquímicos, hizo evidente que no se dominaba el tema y que no existía el hábito de la lectura.

$\mathrm{Al}$ inicio de la intervención se generaron desequilibrios e incertidumbre, lo cual se superó al tomar conciencia de que los conocimientos eran accesibles y comprensibles; así, las siguientes secuencias evidencian cambios favorables en la comprensión y en el logro de aprendizajes con base en el análisis crítico de las situaciones problema.

Los estudiantes al saber que existiría al final de la sesión una explicación teórica accesible y comprensible del tema les dio seguridad y favoreció que se abrieran a la metodología de aprendizaje propuesta para trabajar en las actividades de aprendizaje diseñadas en las secuencias.

Esta fue una devolución adicional muy importante, pues los hábitos de estudio y el trabajo colaborativo se fortalecieron lo mismo que sus aprendizajes (Azorín, 2018). Al final, en las últimas secuencias fue innecesario realizar el "repaso" sobre el tema abordado, pues los estudiantes ya se habían apropiado de él al buscar soluciones a las situaciones problema, incluso resultaba repetitivo y provocaba poco interés, además de distracción. Respecto del trabajo de los estudiantes con las situaciones problema se observó como las desarrollaron en tres momentos: acción, formulación y validación, de acuerdo con la teoría de las situaciones. En dichos momentos de la situación caracterizamos los avances y retrocesos de los estudiantes, en lo individual, en tríada y grupalmente (García, y Posso, 2017).

Se observó la conformación de tres tipos de estudiantes quienes presentaron un desarrollo avanzado, otros de progreso medio y los que avanzaron lo suficiente para demostrar que sí aprendían del tema, cubriendo lo básico solicitado.

El cuestionarse sobre soluciones a problemas de docencia sitúa a los profesores en la posición de investigadores, preguntándose sobre sus marcos teóricos y prácticas cotidianas, convirtiendo así a la investigación-acción en un proceso de aprendizaje que les conduce a realizar análisis críticos de las situaciones que abordan y teorizando sobre sus prácticas. La articulación empleada en este artículo de investigación-formación-acción, implica religar la docencia (formación) y la investigación a través de la acción de los profesores involucrados en procesos formativos y de intervención áulica. 


\section{Conclusiones}

El objetivo de la investigación se alcanzó al lograr que los estudiantes vivenciaran la experiencia de aprendizaje mediante secuencias didácticas, y con ello, fueran capaces de movilizar sus recursos cognitivos ante las situaciones problema que enfrentaron, de manera particular, en las temáticas de la química aplicada al desempeño profesional.

Esta estrategia didáctica para la enseñanza de la química en la educación superior demostró su efectividad en cuanto a los aprendizajes logrados y a la participación de los estudiantes en una nueva metodología que implicó vivir un nuevo contrato didáctico.

El principal detonante para la mejora de los logros académicos son las situaciones problema relevantes, reales y situadas dentro de un contexto profesional que capta el interés de los estudiantes. Además los estudiantes muestran que su nivel de desarrollo del pensamiento crítico aumenta.

Haber trabajado con los estudiantes y seguirlos en la progresión de sus aprendizajes permitió, como lo destaca la teoría, ajustar y refinar la situación didáctica cuando los estudiantes acuden a comportamientos y actitudes que muestran la fuerte dependencia que tienen del profesor, y la nostalgia que sienten por retornar a la comodidad que les brindan las clases tradicionales, lo cual se hizo evidente en nuestra experiencia.

Puede concluirse que las situaciones problema en cuanto a su planeación e implementación muestran su efectividad durante la intervención áulica, donde los estudiantes logran niveles de autonomía, como se describe en en el apartado de fundamentos teóricos.

Las situaciones problema resultan de gran valía al llevar a los estudiantes a adquirir mayor compromiso y más responsabilidad en el trabajo colaborativo. En cuanto al trabajo con situaciones problema es de desatacar que emplea más tiempo que el utilizado en las clases donde priva la clase magistral basada en la enseñanza por temas.

\section{Limitaciones del estudio}

Entre las limitaciones del estudio se mencionan: a) la resistencia al cambio por parte de los estudiantes, ya que se encuentran en una situación cómoda con sus esquemas tradicionales (que han interiorizado durante su vida escolar); b) el nivel de conocimientos previos de los estudiantes al inicio del curso; c) encontrar el balance ideal entre los requerimientos de la metodología en estudio y las ayudas docentes, es decir, aprendizajes por si mismos, clase magistral, y trabajo extraclase; y d) la necesidad de crear conciencia respecto de su capacidad para aprender. 


\section{Líneas de acción futuras}

Como lineas de acción u oportunidades para futuras investigaciones se encuentran: a) Dado su carácter interdisciplinario, aplicar el M-DECA en otras asignaturas sobre todo las relacionadas con ciencias que representan dificultad a los estudiantes; b) En relación con las innovaciones digitales, aplicar el programa Programa Thumult Hype en un curso completo, ya que su empleo en esta investigación generó muy buenos resultados; y c) Crear comunidades de práctica de profesores interesados en mejorar su práctica pedagógica, aplicando el M-DECA.

\section{References:}

1. Alegre-Agís, E. y Riccò I. (2017). Contribuciones literarias, biográficas y autoetnográficas a la antropología médica en España: el caso catalán. Salud Colectiva, 13(2), 279-293. https://doi:10.18294/sc.2017.1203

2. Alvarado, K. W. (2012). Incidencia de los trabajos prácticos en el aprendizaje de los estudiantes de Química General I en conceptos de materia, energía y operaciones básicas, en la UPNFM de la sede de Tegucigalpa. [Disertación de Maestría no publicada]. Universidad Pedagógica Nacional, Francisco Morazán, Tegucigalpa.

3. Anderson, G. y Herr, K. (2007). El docente-investigador: investigación-acción como una forma válida de conocimientos. En I. Sverdlick, (Comp.), La investigación educativa. Una herramienta de conocimiento y acción. Novedades Educativas.

4. Ausubel, D. P., Novak, J. D. y Hanesian, H. (2006). Psicología educativa un punto de vista cognoscitivo. Trillas

5. Azorín-Abellán, C. (2018). El método de aprendizaje cooperativo y su aplicación en las aulas. Perfiles Educativos, 40(161), 181-194. https://doi.org/10.22201/iisue.24486167e.2018.161.58622

6. Batallán, G. (2007). Docentes de infancia. Antropología del trabajo en la escuela primaria. Paidós.

7. Blanchard, M., \& Muzás, M. (2016). Los Proyectos de Aprendizaje: un marco metodológico clave para la innovación. Narcea ediciones.

8. Blanco, M. (2012). ¿Autobiografía o autoetnografía? Desacatos, 38, 169-178.

9. Bolívar, A. y Domingo, J. (2019). La investigación (auto) biográfica y narrativa en educación. Octaedro.

10. Bretz, S.L., Michel Fray, Laura B. Bruck y Marcy H. Towns. (2013). What Faculty Interviews Reveal about Meaningful Learning in the Undergraduate Chemistry Laboratory. J. Chem. Educ, 90(3), 281-288. https://doi.org/10.1021/ed300384r 
11. Brousseau, G. (2007). Iniciación al estudio de la teoría de las situaciones didácticas. Libros del Zorzal.

12. Brown, R. (2015). La evaluación auténtica: El uso de la evaluación para ayudar a los estudiantes a aprender. RELIEVE. Revista Electrónica de Investigación y Evaluación Educativa, 21(2), 1-10. Doi: http://dx.doi.org/10.7203/relieve.21.2.7674

13. Cárdenas, F.A., y Pastrana, L.H. [Editores Académicos]. (2016). Aprendizaje y evaluación auténtica. Experiencias y perspectivas de aplicación. Facultad de ciencias de la educación, maestría en docencia. Universidad La Salle. Kimpres S.A.S.

14. Castillo, A.; Ramírez, M.; González, M. (2013). El aprendizaje significativo de la química: condiciones para lograrlo. Omnia, 2, 1124.

15. Cuenca, C.V. (2011). Propuesta de estrategias de enseñanza para la promoción de la salud desde la Química del Carbono, tecnología y ambiente, Tercer grado de educación secundaria para tres instituciones educativas del país ubicadas en el cono este y sur de la ciudad de Lima y pertenecientes al grupo de escuelas promotoras de la salud. [Disertación de maestría no publicada]. Pontificia Universidad Católica del Perú, Lima.

16. De la Espriella, R., y Gómez, C. (2020). Teoría fundamentada. Revista Colombiana de Psiquiatría, 2, 127-133. DOI: https://doi.org/10.1016/j.rcp.2018.08.002

17. Del Valle, C.M. y Curotto, M. M. (2008). La resolución de problemas como estrategia de enseñanza y aprendizaje. Revista Electrónica de Enseñanza de las Ciencias, 2, 463-479.

18. Díaz-Barriga, F. (2006) Enseñanza Situada: Vínculo entre la escuela y la Vida. Mc Graw Hill.

19. Díaz-Barriga, F. y Hernández, R. G. (2010). Estrategias docentes para un aprendizaje significativo una interpretación constructivista. Mc Graw Hill.

20. Edwards, J., Ceci, Ch., y Ratcliffe, E. (2016). What the public really thinks about chemistry. Chemistry International, 38(3-4), 16-19. Doi: https://doi.org/10.1515/ci-2016-3-406

21. Elliott, J. (2005). La investigación-acción en educación. Ediciones Morata

22. Escribano, E. (2017). La educación en América Latina: desarrollo y perspectivas. Actualidades Investigativas en Educación, 17(2), 1-23. Doi 10.15517/aie.v17i1.28147

23. Fathi-Azar, E., Bayrami, M., Vahedic, S., \& Adli, V. A. (2013). The efect of instructional analogies in interaction with logical thinking 
ability on achievement and attitude toward chemistry. Chem. Educ. Res. Pract., 14(14), 566-575. Doi https://10.1039/c3rp00036b

24. Fernández, J., y Moreno, J. (2008). La química en el aula: entre la ciencia y la magia. Universidad Politécnica de Cartagena (UPCT), Departamento de Ingeniería Química y Ambiental.

25. García, B. E., Posso, L. M. (2017). Situaciones didácticas en la enseñanza del enlace químico. Educere, 21(70), 581-592.

26. Garritz, A. (2010). La enseñanza de la química para la sociedad del siglo XXI, caracterizada por la incertidumbre. Educ. Quim., 21(1), 215.

27. Grijalba, J., Mendoza, \& Beltrán, H. (2020). La formación del pensamiento sociocrítico y sus características: necesidad educativa en Colombia. Universidad y Sociedad, 12(1), 64-72.

28. Guzmán, I., Marín, R., e Inciarte, A. J. (2014). Innovar para transformar la docencia universitaria. Un modelo para la formación por competencias. Ediciones Astro Data, S.A.

29. Habermas, J. (1986). Ciencia y técnica como ideología. Tecnos.

30. Herradón, H. (2012). Enseñanza y Divulgación de la Química y la Física. Garceta.

31. Holbrook, J. (2005). Making Chemistry teaching relevant. Chemical Education International, 6(1), 1-12.

32. Huchim, D. y Reyes, R. (2013). La investigación biográfico-narrativa, una alternativa para el estudio de los docentes. Revista Electrónica Actualidades Investigativas en Educación, 13(3), 1-27.

33. Jonnaert, P., Barrete, J., Masciotra. y Yaya, M. (2008). Las competencias como organizadoras de los programas de formación: hacia un desempeño competente. Profesorado. Revista de curriculum y formación del profesorado, 12(3), 1-31

34. Lazo, L. (2012). Estrategia para la enseñanza y el aprendizaje de la Química General para estudiantes de Primer Año de Universidad. Diálogos Educativos, 23, 68-89.

35. Marín, R., Guzmán, I., Inciarte, A. y Araya, E. (2015). Intervenir e investigar en el aula. Experiencias en la formación de profesores. Alfagrama Ediciones.

36. Martínez, B. M. (2014). Conceptos claves, laboratorios de investigación y bases de datos: estrategias para la enseñanza de Bioquímica en siglo XXI. Química Viva 13(1), 5-17.

37. Matlin, S., Mehta, G., Hopf, H., \& Krief. (2016). One-world chemistry and systems thinking. Nature Chemistry, 8(5), 393-398.

38. Medrano, C. M., Osuna, I., y Garibay, J. L. (2015). La eficiencia del aprendizaje cooperativo en la enseñanza de la química en el nivel 
medio superior. RIDE Revista Iberoamericana para la Investigación y el Desarrollo Educativo, 6(11), 1-10.

39. Menargues, S. y Gómez, S. (2012). Estrategias didácticas para la promoción de la química en la enseñanza secundaria y bachillerato. An. Química, Real Sociedad Española de la Química, 109(3), 218-223.

40. Monereo, C. (2009). La autenticidad de la evaluación. En M. Castelló (ed.) La evaluación auténtica en enseñanza secundaria y universitaria (pp. 15-32). Edebé.

41. Moreira, M. A. (2009). Aprendizaje significativo de las ciencias: Condiciones de ocurrencia, progresividad y criticidad. Ponencia en II Jornadas de Enseñanza e Investigación Educativa en el campo de las Ciencias Exactas y Naturales. La Plata, Argentina. http://www.fuentesmemoria.fahce.unlp.edu.ar/trab_eventos/ev.612/ev .612.pdf

42. Moreira, M. A. (2012). ¿Al final, que es aprendizaje significativo? [Versión electrónica]. Revista Qurriculum, 25, 29-56.

43. Moria, E., \& Zaim, M. (2017). Using Authentic Assessment to Better Facilitate Teaching and Learning: The Case for Students' Writing Assessment. Advances in Social Science, Education and Humanities Research (ASSEHR), 148, 333-337. Doi: 10.2991/icla-17.2018.57

44. Ordaz, G. J., y Britt, M. (2018). Los caminos hacia una enseñanza no tradicional de la química. Actualidades Investigativas en Educación, 18(2), 1-20.

45. Ortega, D.T. y Palacios, J. (2015). Experiencias en la enseñanza experimental basada en competencias. [Versión electrónica]. Educación Química-Universidad Nacional Autónoma de México, 26(1), 31-42.

46. Overton, T., Potter, N. y Leng, C. (2013). A study of approaches to solving open-ended problems in chemistry. Chem. Educ. Res. Pract., 46(14), 468-475. Doi: 10.1039/c3rp00028a

47. Panizza, M. (2003). Conceptos básicos de la teoría de las situaciones didácticas. En M. Panizza, O. Bartolomé, C. Broitman, D. Fregona, y H. Itzcovich, Enseñar matemáticas en nivel inicial y el primer ciclo de la EGB, análisis y propuestas. Paidós

48. Perrenoud, P. (2011). Desarrollar la práctica reflexiva en el oficio de enseñar. Profesionalización y razón pedagógica. Graó.

49. Reibstein, R. (2017). A more ethical chemistry. Current Opinion in Green and Sustainable Chemistry, 8(12), 36-44.

50. Roegiers, X. (2010). Una pedagogía de la integración: competencias e integración de los conocimientos en la enseñanza. Fondo de cultura eonómica. 
51. Roegiers, X. (2016). Marco conceptual para la evaluación de las competencias. Oficina Internacional de Educación de la UNESCO (OIE-UNESCO). http://www.ibe.unesco.org/es/documento/marcoconceptual-para-la-evaluaci\%C3\%B3n-de-competencias

52. San Martín, D. (2014). Teoría fundamentada y Atlas.ti: recursos metodológicos para la investigación educativa. Revista Electrónica de Investigación Educativa, 16(1), 104-122. http://redie.uabc.mx/vol16no1/contenido-sanmartin.html

53. Sarzosa, E. Z. y Pérez, Á. M. (2017). Situaciones didácticas para el aprendizaje de la argumentación en Química. Educere, 21(70), 593610.

54. Soto, M. C. (2015). Experiencias docentes en intervención psicomotriz para el logro de competencias en preescolar (Dissertation doctoral no publicada), Universidad Autónoma de Nuevo León.

55. Suárez, D. y Metzdorff, V. (2018). Narrar la experiencia educativa como formación. La documentación narrativa y el desarrollo profesional de los docentes, en Espacios en Blanco. Revista de Educación, 28, 49-74.

56. Zambrano, A. (2019). Naturaleza y diferenciación del saber pedagógico y didáctico. Pedagogía y Saberes, 50, 75-84. 